\title{
Agricultural probiotics enter spotlight
}

In a whirl of announcements, several companies declared their overlapping interests in developing microbial-based products to change plant performance in agriculture without genetic manipulation. Last November, Flagship VentureLabs of Cambridge, Massachusetts, unveiled Symbiota, also of Cambridge, with news that candidate products are being developed for crops such as corn, soy, wheat and cotton, supported by $\$ 7.5$ million in first-round financing. This January, the BioAg Alliance, a collaboration between Monsanto of St. Louis and Novozymes in Copenhagen (Nat. Biotechnol. 32, 211, 2014), reported promising yield improvements following full-year field trials in which microbial strains were applied to corn and soybeans.

Agbiotech companies are embracing the plant microbiome with the same enthusiasm with which many medical researchers pursue the human microbiome. "This is a hot topic right now, with lots of private and public funding being pumped into it, mainly driven by concerns over crop diseases, climate change and increased yield needs for a growing human population," says Jack Gilbert of Argonne National Laboratory in Lemont, Illinois, and the University of Chicago, articulating the issues facing plant breeders, which researchers and firms are tackling through various routes (Box 1). Gilbert also manages the Earth Microbiome Project, an ongoing effort to characterize microbial diversity.

"These technologies have significant potential to ... help farmers produce more crops with fewer inputs," says Colin Bletsky of Novozymes. "For microbial performance, which typically stands out under more stressed conditions, this

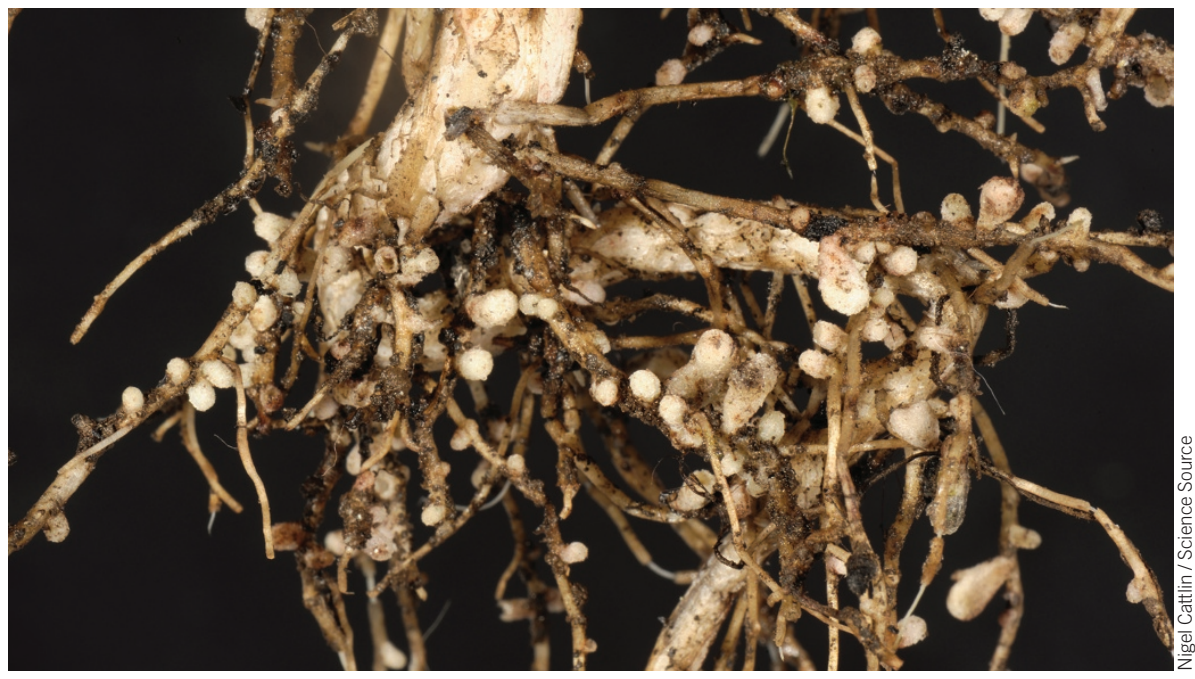

Nitrogen-fixing rhizobia, soil bacteria, live in a symbiotic relationship with legumes, once they become established within root nodules.

type of benefit in favorable growing conditions is very promising," he adds.

Several startup companies including about a half-dozen dedicated US companies as well as others in Canada, Spain and New Zealand are seeking to leverage the microbiomes of agricultural and ornamental plants for commercial purposes. Symbiota's new executive chairman Robert Berendes, formerly with Syngenta, says the company aims to develop "a step change in crop performance" through symbiosis with plants rather than genetic manipulations. Other companies with similar goals include BioConsortia of Davis, California, which raised \$15 million last year; AgBiome of Research Triangle Park, North Carolina, which raised $\$ 17.5$ million late last year from Syngenta of

\section{Box 1 Stored germplasm consortium to improve crops}

An international consortium, calling itself Diversity Seek, or DivSeek, in January launched efforts to "unlock crop diversity" by extracting genetic information from some seven million samples of crop varieties and wild relatives stored in gene banks. The overall aim is to accelerate development of "climate-ready, high-yielding and nutritious crops." It is intended "to bridge the gap between the information requirements of gene bank curators, plant breeders and more targeted upstream biological researchers."

As part of its formal launch in San Diego, DivSeek brought together 69 founding organizations, including the Global Crop Diversity Trust, the CCGIAR Consortium, the International Treaty on Plant Genetic Resources for Food and Agriculture and the Global Plant Council-all with worthy credentials in global agriculture. They are already working with a white paper from early 2014 outlining DivSeek's near-term activities along with its longer-term goals.

"This first year is very much about scoping out what is needed first, before pilot projects and case studies are carried out," says Paul Gough of the Global Crop Diversity Trust in London, a spokesman for DivSeek. Early tasks include developing "a system of internationally agreed-upon standards, protocols, tools, resources and best practices" before building an information platform providing access to data describing crop germplasm held in gene banks as well as "digital genomes." nearby Greensboro, Monsanto and Novozymes; and Adaptive Symbiotic Technologies of Seattle. Yet another potential rival in this sector, Agradis of La Jolla, California, was gobbled up by Monsanto early in 2013. Similar efforts outside the US include BioDiscovery in Parnell, New Zealand, which is allied with BioConsortia; Mycovitro in Granada, Spain; and Onocucor in Montreal, Quebec, Canada.

Although some companies pursue many crops and others focus on only a few, their strategies overlap considerably. Most are seeking potentially beneficial microorganisms that associate with plants-sometimes with root systems and surrounding soils, and in other instances, with leaf surfaces or internal tissues-that then can play useful roles. Once identified, those microorganisms are to be harnessed as 'probiotics' and mainly used in mixtures rather than handled one microbial species at a time; they'll be applied in ways that induce augmented responses to fertilizers, tolerance to conditions such as drought or salinity, resistance to pathogen-borne diseases, or in other microbes will be used to treat the maturing plants, in other cases, the seeds.

Many efforts to develop probiotics for plants "show great promise," Gilbert says, but "only in very few cases have the key experiments been done to demonstrate that application of a key biological agent has a specific effect on the crop itself." He anticipates someone claiming success with a plant probiotic within the next two years, but says that "the wide production of probiotics to solve ills is probably a long way off, an estimated 10 to 15 years."

Jeffrey L Fox Washington, DC ways that improve yields. In some cases, the 